\title{
Delayed Cerebrospinal Fluid Leakage 10 Years After Transsphenoidal Surgery and Gamma Knife Surgery
}

\author{
-Case Report-
}

\author{
Yoshikazu OGAWA and Teiji TOMINAGA*
}

\begin{abstract}
Department of Neurosurgery, Kohnan Hospital, Sendai, Miyagi; *Department of Neurosurgery, Tohoku Graduate School of Medicine, Sendai, Miyagi
\end{abstract}

\begin{abstract}
A 38-year-old woman presented with repeated episodes of meningitis. She had undergone transsphenoidal tumor removal followed by gamma knife irradiation in 1994. Complete remission was achieved. Intermittent cerebrospinal fluid (CSF) leakage began in 2004, and transsphenoidal surgery was performed for direct repair of the skull base defect. Operative findings showed that the sellar floor was uncovered, and CSF continuously escaped through the cyanoacrylate polymer framework of the previous repair. Reconstruction used autologous muscle pieces and cyanoacrylate polymer adhesive. The CSF leakage was presumably due to delayed radiation damage to the mucous membrane of the skull base. Several methods for reconstruction of the sellar floor have been proposed, which all rely on tissue regeneration including the arachnoid, dura mater, and mucus membrane of the sphenoidal sinus. Preservation of the arachnoid membrane and minimizing removal of the mucous membrane are essential, especially if postoperative irradiation is anticipated.
\end{abstract}

Key words: cerebrospinal fluid leakage, transsphenoidal surgery, radiosurgery

\section{Introduction}

The transsphenoidal approach is useful not only for tumors which arise from intrasellar tissues but also for $20 \%$ of brain tumors. The usual targets are histological benign tumors like pituitary adenoma and craniopharyngioma. Advances in surgical technique and accumulation of experience have improved the prognosis, and recently the operative field has been widened by methods such as the extended transsphenoidal approach. ${ }^{1)}$ Several methods for reconstruction of the sellar floor have been proposed, which all rely on tissue regeneration including the arachnoid, dura mater, and mucus membrane of the sphenoidal sinus. One important complication of the transsphenoidal approach is cerebrospinal fluid (CSF) leakage, which occurs in $0.4 \%$ to $9 \%$ of cases. ${ }^{3)}$ This complication will only occur if the arachnoid membrane and skull base structure are simultaneously disrupted.

We treated a patient with delayed CSF leakage which occurred 10 years after the initial treatment for pituitary adenoma by transsphenoidal surgery

Received March 8, 2007; Accepted August 7, 2007 and postoperative gamma knife radiosurgery.

\section{Case Report}

A 35-year-old woman presented at our outpatient department with bacterial meningitis in August 2004. She had developed secondary amenorrhea at the age of 22 years due to prolactin-secreting pituitary adenoma, and was initially medicated with bromocriptine. However, the serum prolactin level decreased only to $180 \mathrm{ng} / \mathrm{ml}$. Magnetic resonance (MR) imaging showed a tumor in the sella turcica of $10 \mathrm{~mm}$ diameter which had invaded the right cavernous sinus.

She was introduced to our outpatient department, and transsphenoidal surgery was performed at Tohoku University Hospital in 1994. Subtotal removal of the tumor was achieved. After 6 months of observation, gamma knife radiosurgery was performed with isocenter dose of $35.7 \mathrm{~Gy}$ and marginal dose of 70\% to 90\% (Fig. 1). Follow-up MR imaging in 1996 revealed significant tumor shrinkage, thinning of the normal pituitary tissues, and disappearance of packed tissues in the sphenoidal sinus (Fig. 2A). Finally, complete remission of the tumor was 

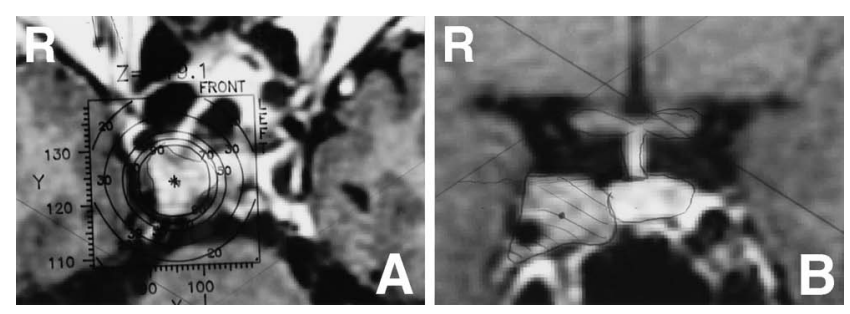

Fig. 1 A: Axial $T_{1}$-weighted magnetic resonance (MR) image with contrast medium indicating dose planning of gamma knife surgery. Note that the $70-90 \%$ isodose center covered the sellar floor which corresponded to the point of cerebrospinal fluid leakage. B: Reconstructed coronal $T_{1}$-weighted $M R$ image with contrast medium from thin axial slices indicating dose planning of gamma knife surgery. Note that the shaded portion indicates the $70-90 \%$ isodose center.
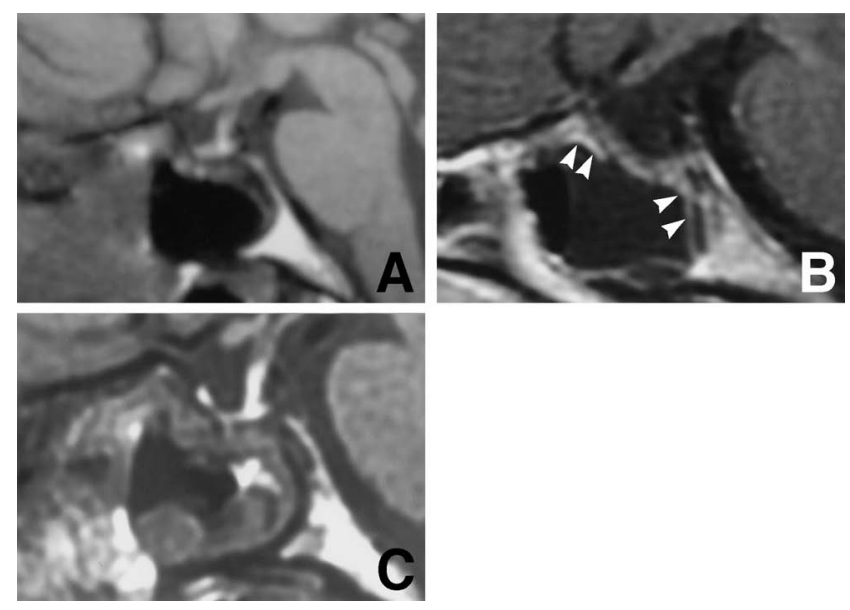

Fig. 2 A: Sagittal $T_{1}$-weighted magnetic resonance (MR) image in 1996 revealing thinning of the normal pituitary tissues, and disappearance of packed tissues in the sphenoidal sinus. B: Preoperative sagittal $T_{1}$-weighted MR image in 2005 revealing disruption of the mucus membrane of the sellar floor (arrowheads) and a fluid-fluid level in the sphenoidal sinus, isointense to the cerebrospinal fluid. C: Sagittal $\mathbf{T}_{1}$-weighted MR image in 2007 revealing continuous mucus membrane of the sellar floor and dissolution of packed tissues in the sphenoidal sinus.

achieved. Serum prolactin level decreased to 45.0 $\mathrm{ng} / \mathrm{ml}$ at that time.

She later married and had a child. She began to feel an odd sensation that liquid was always flying into her pharynx in spring 2004. She suffered from
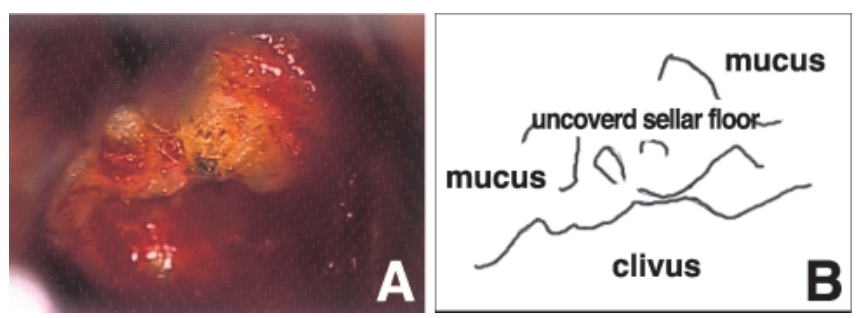

Fig. 3 A: Intraoperative photograph showing the sellar floor not covered by the mucous membrane. Cerebrospinal fluid continuously escaped through the adherent cyanoacrylate polymer framework. B: Schema of intraoperative findings.

repeated episodes of bacterial meningitis, and was treated by intravenous administration of antibiotics with bed rest in August and September 2004. About one month later, she had recovered with no signs of inflammation. A third episode of meningitis occurred in April 2005. After recovery from the inflammation, she was discharged on foot with prophylactic antibiotic medication. She was again admitted for direct repair surgery of the skull base in October 2005. Head MR imaging showed a fluidfluid level in the sphenoidal sinus, and disruption of the mucus membrane of the sellar floor but without signs of tumor recurrence (Fig. 2B). Investigation of pituitary, thyroid, and adrenal hormones showed low levels of growth hormone and luteinizing hormone, but all other hormones were within the normal ranges including $31.9 \mathrm{ng} / \mathrm{ml}$ prolactin.

A speculum was inserted sublabially and between the nasal septa to reach the anterior wall of the sphenoidal sinus, which was replaced by thick granulation. After removal of this granulation, the sphenoidal sinus was exposed, which was filled with transparent CSF. The mucous membrane of the skull base was completely thinned, and the sellar floor was uncovered. The muscle pieces which had been used to entirely cover the sellar floor were dissolved, and only a framework of cyanoacrylate polymer adhesive remained. CSF continuously escaped through this structure (Fig. 3). Autologous muscle pieces were again attached with cyanoacrylate polymer adhesive to cover the whole of the sellar floor, then the patient was placed in the head down position for one minute to ensure waterproof skull base reconstruction. Fibrin glue was then spread followed by packing of the sphenoidal sinus with autologous lipid.

Immediately after the operation, the odd sensation of liquid in the pharynx disappeared. Head MR imaging revealed no signs of fluid collection in the 
sphenoidal sinus. Twelve days after the operation, she was discharged on foot with prophylactic antibiotic medication. No signs of meningitis or fluid collection in the sphenoidal sinus were observed in January 2007. MR imaging showed continuous and swollen mucus membrane of the sellar floor, and dissolution of the packed tissues in the sphenoidal sinus (Fig. 2C).

\section{Discussion}

The most common methods for preventing postoperative CSF leakage after transsphenoidal surgery are autologous bone plate placement in the epidural space, and packing of the sella turcica and/or sphenoidal sinus with autologous lipid, fibrin glue, and gelatin foam. ${ }^{2,4,5,7)}$ Spinal drainage has also been recommended for several days after the operation. ${ }^{6}$ However, all these methods rely on the expectation of biological membrane regeneration, as these repair materials function only temporarily and are finally dissolved in the body. In the healing stage, the arachnoid membrane, dura mater, and mucous membrane of the sphenoidal sinus can all prevent CSF leakage. In our patient, all three of these barriers had become disrupted. In general, mucous membrane of the sphenoidal sinus is aggressively removed to perform the operation in a dry field and to avoid postoperative formation of mucocele. After tumor removal, the sellar floor is repaired and the sphenoidal sinus is fully packed with autologous fat or other materials. In our institution, the mucous membrane of the sphenoidal sinus has been sharply incised and furled up like a curtain since June 2005. After tumor removal, the sellar floor is repaired with autologous bone plate and muscle pieces, and the mucous membrane drawn down to cover the sellar floor. Since then 183 patients have been treated and no severe mucocele has been found.

Gamma knife therapy leads to cure of pituitary adenoma, but may also disrupt the regenerated mucous membrane of the sphenoidal sinus. Therefore, surgical injury to the sphenoidal sinus mucous membrane should be minimized, and the arachnoid plane preserved, especially if postoperative irradiation is anticipated. The arachnoid membrane is sometimes intentionally incised during extended trans- sphenoidal surgery, and the tumor is removed through a CSF space such as the prechiasmatic cistern. However, arachnoid membrane preservation should be the principle at standard transsphenoidal surgery, and excessive removal of the mucous membrane of the sphenoidal sinus should be avoided.

\section{References}

1) Kitano M, Taneda $M$ : Subdural patch graft technique for watertight closure of large dural defects in extended transsphenoidal surgery. Neurosurgery 54: 653661,2004

2) Kudo H, Sakagami Y, Kawamura A, Tamaki N: Delayed cerebrospinal fluid rhinorrhea seven months after transsphenoidal surgery for pituitary adenoma — case report. Neurol Med Chir (Tokyo) 40: 160-163, 2000

3) Laws ER Jr, Thapar K: Surgical management of pituitary adenomas. Baillieres Clin Endocrinol Metab 9: 391-405, 1995

4) Saeki N, Tokunaga H, Hoshi S, Sunami S, Sunami K, Uchino F, Yamaura A: Delayed postoperative CSF rhinorrhea of intrasellar arachnoid cyst. Acta Neurochir (Wien) 141: 165-169, 1999

5) Seiler RW, Mariani L: Sellar reconstruction with resorbable vicryl patches, gelatin foam, and fibrin glue in transsphenoidal surgery: a 10-year experience with 376 patients. J Neurosurg 93: 762-765, 2000

6) van Aken MO, Feelders RA, de Marie S, van de Berge JH, Dallenga AH, Delwel EJ, Poublon RM, Romijn JA, van der Lely AJ, Lamberts SW, de Herder WW: Cerebrospinal fluid leakage during transsphenoidal surgery: postoperative external lumbar drainage reduces the risk for meningitis. Pituitary 7: 89-93, 2004

7) Van Velthoven V, Clarici G, Auer LM: Fibrin tissue adhesive sealant for the prevention of CSF leakage following transsphenoidal microsurgery. Acta Neurochir (Wien) 109: 26-29, 1991

Address reprint requests to: Yoshikazu Ogawa, M.D., Department of Neurosurgery, Kohnan Hospital, 4-20-1 Nagamachiminami, Taihaku-ku, Sendai, Miyagi 982-8523, Japan.

e-mail: yogawa@kohnan-sendai.or.jp 